\title{
El proyecto de Escuela Única de Pedagogía: demandas y propuestas en torno a la formación unificada de profesores en Chile, 1928-1990
}

School United of Pedagogy Project:

Demands and Proposals around the Unified Teachers Training

in Chile, 1928-1990

\section{Camila Pérez-Navarro}

Facultad de Educación, Pontificia Universidad de Chile

camila.perez@uc.cl

\section{Resumen}

\begin{abstract}
Este trabajo analiza los distintos proyectos de unificación del sistema de formación de profesores en Chile, iniciativa fuertemente demandada por los gremios magisteriales a lo largo del siglo Xx. Mediante el análisis de fuentes primarias, tales como escritos de líderes gremiales, documentos legislativos, actas de congresos pedagógicos, informes gubernamentales y propuestas magisteriales de las diferentes épocas estudiadas, se reconstruye la historia larga de la demanda de unificación del sistema nacional de formación docente, exponiendo las ideas que lo sustentaron y los elementos que lo conformaron. Específicamente, la investigación responde a la interrogante de cómo el proyecto de unificación, planteado inicialmente en 1928, subsistió en el ideario magisterial hasta las décadas de 1960 y 1970, y en qué medida la propuesta logró imponerse durante el periodo de la dictadura y en el actual modelo de formación Inicial de docentes en Chile.
\end{abstract}

Palabras clave: Escuela Única de Pedagogía, formación de profesores, Chile.

${ }^{1}$ La autora agradece a Iván Núñez Prieto por facilitarle las fuentes y la documentación para realizar este trabajo, así como a los dos evaluadores que enriquecieron este artículo con sus valiosos comentarios. 


\begin{abstract}
This paper analyzes severals projects addressed to unify the Teacher Training in Chile, an initiative strongly demanded by the teachers' unions throughout the twentieth century. By analyzing multiple sources such as: documents' union leaders, legislative documents, teaching centers records, government reports as well as proposals from the years examined in this paper, I trace the long history for demands of unification of the national training teacher's system, introducing the ideas behind this project and the elements that made It possible. This paper seeks to shed new light over the question on how this unification project, originally born in 1928, managed to survive in the 1960s-70s, and in what extent the current Teacher Training was informed by the dictatorship.
\end{abstract}

Keywords: School United of Pedagogy, Unified Teachers Training in Chile, Chile.

\title{
Introducción: la histórica parcelación en el sistema de formación de profesores en Chile
}

La presente investigación constituye una puesta en valor del histórico proyecto de Escuela Única de Pedagogía. A lo largo del siglo xx, la fundación de un único sistema de formación de profesores se presentó como una aspiración transversal de los gremios magisteriales, siendo un componente fundamental de los distintos proyectos de reforma educativa de la historia de la educación chilena. Esta demanda de unificación se sustentó en la paulatina masificación del sistema nacional de educación durante los siglos XIX y XX, la cual implicó una constante y creciente diversificación del sistema de formación de profesores.

En este marco, varias instituciones preparaban maestros y docentes de "distinta categoría": por un lado, los maestros primarios se formaban en escuelas normales, instituciones que principalmente entregaban a sus aprendices conocimientos pedagógicos que eran complementados con los saberes emanados de las prácticas en aula; mientras que, por otro, los profesores de educación secundaria egresaban del Instituto Pedagógico, con saberes técnicos y disciplinarios mucho más especializados (Cox y Gysling, 1990).

Esta diferencia se profundizó por la preparación de los profesores que se desempeñarían en escuelas técnicas, establecimientos industriales e institutos comerciales, quienes se formaban en el Instituto Pedagógico Técnico. De esta manera, mientras la formación de los maestros primarios de las escuelas normales era controlada directamente por el Ministerio de Educación Pública; la formación de los profesores secundarios estuvo a cargo de la Universidad de Chile por más de 100 años.

Este escenario se complejizó en la mitad del siglo xx. La multiplicación de instituciones formadoras de docentes a nivel territorial a partir de los años 50 -dependientes, principal- 
mente, de la Rectoría de la Universidad de Chile, de la Universidad Católica y de la Universidad de Concepción - demuestra la alta diversificación alcanzada por el sistema; ${ }^{2}$ como ejemplo representativo se puede exponer la creación, a principios de la década de 1960, de los colegios universitarios regionales (como dependencias institucionales de la Universidad de Chile), los cuales impartieron carreras cortas de pedagogías conducentes a los títulos de profesor de educación primaria en ciudades como Antofagasta, La Serena, Chillán, Osorno y Temuco.

De esta manera, en 1950 se consolidó una parcelación institucional en la formación de los profesores (los estudiantes de pedagogía recibían distintos saberes y títulos), lo cual era visto por los actores de la época como uno de los factores de crisis educativa. En este marco, todas las iniciativas y los proyectos de transformación que se examinarán en este artículo plantearon que la unificación de la formación de profesores contribuiría a solucionar la crisis endémica de la educación chilena, ${ }^{3}$ porque permitiría superar la desvinculación existente entre los niveles de enseñanza del sistema educativo (principalmente entre primaria y secundaria), la desigual formación pedagógica recibida por maestros de primaria y profesores de enseñanza secundaria, las diferencias entre el tipo de profesor que atendía a cada nivel educacional, y la calidad de la educación que se impartía en las escuelas del país. Estos argumentos, planteados por los actores educativos, se presentarán a lo largo de este artículo.

La presente investigación tiene como objetivo analizar las demandas de unificación de la formación de profesores en Chile, así como reflexionar en torno a los alcances de las propuestas promovidas por los maestros durante los procesos de cambio en educación, específicamente, se responde a la interrogante de cómo esta iniciativa, planteada inicialmente

2 Esta diversificación institucional y la inexistencia de una institución que monopolizara la formación del profesorado también se evidencian en la historia de los sistemas de formación docente de países latinoamericanos; por ejemplo, en Argentina, las maestras jardineras (educadoras de párvulos) y las maestras de primaria se formaban en escuelas normales; mientras que la preparación de docentes de nivel medio se realizaba en institutos de formación docente, universidades pedagógicas y facultades de educación —existiendo una gran variedad en los títulos entregados. Un análisis histórico comparativo muestra que durante el siglo xx paulatinamente se unificó la formación docente en países latinoamericanos, desapareciendo la parcelación entre la formación de maestros de nivel primario y profesores de nivel secundario. De acuerdo con Salgado (2006: 34), en el caso de Chile, las razones de unificación fueron de tipo político, desmovilizar políticamente a los docentes y estudiantes de escuelas normales; en El Salvador, de tipo económico, fusionar presupuestos de escuelas normales y universidades;, mientras que "en el resto de países [sic], donde los argumentos políticos no han sido determinantes, aparecen otras razones como, mejorar la formación científica y de investigación muy pobre en la educación normal, atender la educación básica de 9 o 12 años".

${ }^{3}$ Durante la primera mitad del siglo xx, diversos estudios constataron la situación de crisis en la que estaba sumida la educación chilena. Estas investigaciones dieron cuenta de la falta de matrículas y locales escolares, las altas cifras de analfabetismo de la población, el carácter aristocratizante del currículum escolar (contenidos extensos y de tendencia intelectualista), la selectividad y la segmentación social de la educación, y una profunda desorganización en la administración del sistema escolar; entre éstas destacan las de Darío Salas (1913 y 1917), Luis Galdames (1912 y 1913), Víctor Troncoso (1927 y 1954), Víctor Troncoso y Juan Sandoval (1954), Humberto Vivanco Mora (1953), Jorge Ahumada (1958 y1966) y Eduardo Hamuy (1960 y 1961). 
en 1928, subsistió en el ideario magisterial hasta las décadas de 1960 y 1970, y en qué medida la propuesta logró imponerse, por un tiempo, en la formación inicial de docentes, paradójicamente, durante la dictadura cívico-militar liderada por Augusto Pinochet (1973-1990).

A nivel metodológico, la investigación se sustenta fundamentalmente en el análisis de un corpus de fuentes primarias, tales como escritos de líderes gremiales, documentos legislativos, informes gubernamentales y propuestas magisteriales de las diferentes épocas estudiadas, así como en material hemerográfico. Asimismo, el análisis de actas de congresos y jornadas del profesorado relativas a la temática permitió analizar los discursos que circuIaron en torno al proyecto. Con base en este conjunto documental, se reconstruye la historia del proyecto de unificación del sistema de formación docente, exponiendo las ideas que cimentaron cada propuesta y los elementos que caracterizaron los distintos proyectos entre 1928 y 1990.

\section{Las primeras ideas de unificación: las escuelas de profesores en el reformismo de $1928^{4}$}

Durante las tres primeras décadas del siglo Xx, y frente a la realidad educativa nacional, ${ }^{5}$ la formación de profesores fue cuestionada seriamente por las organizaciones magisteriales. El autoritarismo y formalismo de las escuelas normales, "sumado a la incidencia de una ideología social-libertaria que irradió desde la Federación de Estudiantes de Chile de 1920" (Núñez, 2002: 25), impulsó a los maestros reformistas —agrupados en la Asociación General de Profesores de Chile (ACP) ${ }^{6}$ - a intentar destruir los cimientos del normalismo y de la educa-

${ }^{4}$ Respecto a la situación previa a la reforma, a la crisis y a la contrarreforma suscitadas en la dictadura del general, Carlos Ibáñez del Campo, y al rol del movimiento magisterial en el intento de transformación educativa, véase Núñez, Iván (1986) y Reyes, Leonora (2010).

${ }^{5}$ La reforma educativa de 1928 fue una respuesta a la crisis educativa evidenciada por pedagogos y educacionistas como Darío Salas, Enrique Molina o Luis Galdames; implementada en diciembre de 1927 hasta septiembre de 1928, contempló la transformación de todos los niveles educativos y de las prácticas pedagógicas, con el propósito de poner a los estudiantes en el centro del quehacer pedagógico. En septiembre de 1928, el presidente, Carlos Ibáñez del Campo, interrumpió la implementación de la reforma, debido a la tensión generada en los sectores más tradicionales por su radicalidad y por el gasto fiscal que había significado su aplicación; por estas razones, se derogó el Decreto 7.500, se inició la contrarreforma, y se despidió y exoneró a los maestros de la ACP.

${ }^{6}$ Fue una agrupación de maestros que impulsó una propuesta de reforma educacional radical entre 1925 y 1928 , la cual planteaba una completa renovación de las bases de la política educativa nacional a partir de las ideas del movimiento pedagógico de la escuela nueva. De acuerdo con Iván Núñez, la AGP tuvo una orientación política e ideológica socializante y libertaria; sin embargo, "el ideario de la vanguardia de los maestros no era estrictamente anarquista, ni menos marxista o comunista" (1986: 61). Tal como rescata Núñez (1986: 61) en su texto, uno de los líderes de la Asociación explicaba que "en el fondo sustenta una nueva política, pero sin perfiles doctrinarios claros. No se confunde 
ción tradicional, ${ }^{7}$ para establecer una nueva institucionalidad que se responsabilizara de la formación de profesores de manera unificada y especializada, acorde con los principios de la escuela nueva (Serrano et al., 2013). Esta primera propuesta de reestructuración del sistema de formación de profesores era consistente con el proyecto de reforma de la educación nacional, el cual tenía como objetivo principal transformar el sistema educativo en un todo orgánico y funcional que permitiera el desarrollo integral de niños y jóvenes. De acuerdo con el líder gremial, Víctor Troncoso (1925: 33-34):

Sentada la premisa de que el sistema educacional debe ser uno solo, se desprende lógicamente que el profesor de cualquiera rama de la educación debe ser también uno (...) todos los profesores, de cualquiera especialidad y de cualquier grado de la enseñanza deben pasar por la escuela secundaria (actuales liceos); terminados sus estudios humanistas, pasar por la Universidad a profundizar la especialidad de la enseñanza que esté de acuerdo con su vocación y son sus deseos.

Según este precepto, el proyecto de reforma educativa propuesto por la AGP en 1927 planteó el reemplazo del sistema de formación de profesores existente, con el objetivo de avanzar hacia el cumplimiento de los objetivos educativos de la escuela nueva y mejorar la calidad de los estudios pedagógicos (Núñez, 1987). De esta manera, el Decreto con Fuerza de Ley que dio inicio a la reforma educacional establecía que la tarea pedagógica debía ser ejercida por "profesionales que se considerarán actuantes en un mismo proceso y será organizada como una sola unidad funcional en que la enseñanza se inicie, continúe y termine en armonía gradual con el desenvolvimiento del niño, del adolescente y del joven" (Decreto № 7.500, 1927: 1).

Para lograr este cometido, las autoridades educativas establecieron durante el proceso de reforma educacional la supresión de escuelas normales y la creación de escuelas de pedagogía que formaran unificadamente a profesores de primaria y secundaria. De acuerdo con lo señalado en el Decreto N 7.500, del 27 de diciembre de 1927, se reglamentaba que "el Estado asegurará la finalidad de la función educativa formando el magisterio nacional en las escuelas de pedagogía y en los institutos de las universidades, en conformidad a lo que dispongan los reglamentos respectivos".

Dos meses después, esta medida se complementó con la promulgación del Decreto $\mathrm{N}^{\circ}$ 332, en donde se determinaba la creación y el funcionamiento de las escuelas de profesores

con el anarquismo porque ella (la AGP) predica una nueva organización del Estado, de manera que sean los servidores de cada función los que resuelvan sus propios problemas".

${ }^{7}$ La Asociación criticó la figura del maestro tradicional, quien entregaba a sus alumnos saberes de tipo enciclopédico, de forma rutinaria y a través de metodologías que priorizaban la repetición y la memorización de contenidos. 
primarios $^{8}$ en reemplazo de las antiguas escuelas normales, y con la decisión de convertir el Instituto Pedagógico de la Universidad de Chile en una escuela de profesores secundarios, institución que se encargaría de la "formación pedagógica de los docentes de las disciplinas científicas, humanísticas, artísticas y de educación física y manual en la enseñanza secundaria" (Núñez, 1987: 104). A juicio de los profesores comprometidos con la reforma, esta ruptura significaría el fin de la profunda parcelación de las instituciones formadoras de profesores, porque permitiría una continuidad entre las preparaciones pedagógicas de maestros primarios y profesores de educación secundaria, mediante la homogeneización de criterios de formación docente.

La importancia del cambio establecido por el decreto de reforma educacional no sólo tuvo relación con la reestructuración institucional del sistema de formación de maestros, sino con el intento de profesionalización del trabajo docente. La aprobación de reglamentos para la educación primaria y secundaria durante 1928, complementarios al Decreto N 7.500 , definieron los ámbitos que se reformarían en materia de preparación del magisterio. De acuerdo con estas ordenanzas, las escuelas de profesores primarios permitirían el ingreso sólo de estudiantes que hubiesen "rendido a lo menos el $4^{\circ}$ año de Liceo, pero sólo ingresarán al $5^{\circ}$ año aquellos que hayan terminado satisfactoriamente sus estudios secundarios" (Decreto N 332, 1928: 2); mientras que las escuelas de profesores secundarios sólo aceptarían a los postulantes que hubiesen completado rendido el bachillerato. Asimismo, el Reglamento General de Educación Primaria establecía el funcionamiento de escuelas primarias de aplicación, la cuales estaban destinadas a la experimentación mediante la práctica pedagógica de los futuros maestros. Según el planteamiento del historiador, Iván Núñez (1987), estas medidas elevarían el nivel de preparación de los futuros maestros, al imprimírseles a las escuelas de formación pedagógica un carácter profesionalizante.

Sin embargo, estos decretos — que hicieron realidad, por algunos meses, la implementación de la primera demanda de unificación en la formación del profesorado - en la práctica significaron un conflicto mayor en la administración del sistema, porque emergieron disputas en torno a la supresión de las escuelas de origen de los maestros reformistas. Por otro lado, el contexto de crisis $^{9}$ suscitado en el marco de la implementación del modelo radical de reforma educacional implicó la derogación de la legislación elaborada por la AGP, interrumpiéndose el proceso de transformación.

En el proceso de contrarreforma, impuesta por el general Ibáñez, en octubre de 1928, se promulgaron nuevos decretos que determinaban el restablecimiento del Instituto Pedagógico

\footnotetext{
${ }^{8}$ Es fundamental señalar que el decreto concentró las antiguas escuelas normales, y estableció que "las vacantes que se produzcan en el alumnado podrán ser llenadas por aspirantes que hayan rendido a lo menos el $4^{\circ}$ año de Liceo, pero sólo ingresarán al $5^{\circ}$ año aquéllos que hayan terminado satisfactoriamente sus estudios secundarios".

${ }^{9} \mathrm{Al}$ respecto, veáse la nota al pie núm. 4 .
} 
como institución formadora de profesores secundarios y la reorganización de la enseñanza normal, teniendo como base la consideración de "que las Escuelas de Profesores Primarios en su actual organización no satisfacen los propósitos del Gobierno de procurar a la enseñanza nacional un profesorado competente y de elevado espíritu cívico" (Decreto N 5.280, 1928: 1).

Esta reorganización de la enseñanza normal implicó la reposición de los antiguos requisitos de ingreso a las instituciones formadoras de profesores, estableciéndose que para "ingresar a una Escuela Normal Rural se requiere haber terminado con éxito por lo menos el $6^{\circ}$ año de enseñanza primaria" (Decreto $N^{0}$ 5.857, 1928: 1); mientras que para ingresar a una escuela normal urbana se solicitaba haber terminado el primer ciclo de la enseñanza secundaria.

En este nuevo marco, la Universidad de Chile - representada en la figura del profesor, Luis Tirapegui- fue la institución responsable del proceso de reorganización, lo cual mantendría por algún tiempo la centralización administrativa del sistema de formación de profesores en el país. No obstante, la creación de la División General de Educación Primaria en el Ministerio de Educación Pública en 1929, mediante la publicación de una nueva Ley de Educación Primaria Obligatoria (Serrano, Rengifo y Ponce de León, 2012), y la reposición de la dependencia ministerial de las escuelas normales (Decreto $N^{\circ} 220$, 1932) significaron el retorno de la parcelación en la preparación de maestros primarios y secundarios.

\section{La consolidación de la idea de unificación: de las escuelas zonales de pedagogía a la Escuela Única de Pedagogía, 1944-1954}

A pesar de las consecuencias educativas de la contrarreforma implementada en el gobierno de Carlos Ibáñez del Campo (1927-1931), la propuesta de formación unificada de profesores continuó latente en el pensamiento magisterial reformista y se consolidó en los proyectos de transformación educativa elaborados durante las décadas de 1940 y 1950.

Un segundo momento en el que la idea de unificación de la formación de profesores se planteó como componente de los proyectos magisteriales de reforma puede verse en la propuesta de Ley Orgánica de Servicios Educacionales, elaborada durante el gobierno del radical, Juan Antonio Ríos (1942-1946). ${ }^{10}$ De acuerdo con lo evidenciado por Myriam Zemelman, en la década de 1940, la crisis educacional diagnosticada en 1928 persistía; si

\footnotetext{
${ }^{10}$ Durante el gobierno de Juan Antonio Ríos se enfrentaron —encarnados en sus ministros de educación - dos visiones de reforma: mientras que Benjamín Claro Velasco apostó por el diseño y la aplicación de una reforma educacional de carácter integral y radical; el ministro, Enrique Marshall, aplicó un plan de reforma gradual, parcial y experimental. De acuerdo con Zemelman (2010: 53), "Para los economistas, el proceso de industrialización que comenzaba, requería de técnicos y obreros especializados y una población rural capacitada que pudiera ser integrada al nuevo modelo de desarrollo que se intentaba implementar. En cambio, para los pedagogos reformistas, urgía una reforma global de todo el sistema educativo nacional"
} 
bien se habían aumentado las matrículas escolares, había mucha inasistencia escolar; el currículum escolar no respondía a las necesidades de la sociedad, y la fuerte desvinculación entre los niveles educativos continuaba; por ello, para los pedagogos reformistas, era fundamental que se aplicara una reforma en todo el sistema educacional que sustituyera la pedagogía tradicional. "Se señalaba, asimismo, que se hacía necesario solucionar la descoordinación interna del sistema educativo, con el fin de armonizarlo posteriormente, con el sistema productivo" (Zemelman, 2010: 53). Por estas razones, en 1944, el ministro de educación pública, Benjamín Claro Velasco, pidió a los líderes reformistas de 1928, Víctor Troncoso y Daniel Navea, la revisión de un nuevo proyecto de reforma educacional que se presentó al Congreso Nacional. En éste, se planteaba la reorganización del sistema de educación pública en "dos servicios debidamente coordinados en sus fines y planes de acción" (Troncoso y Navea, 1947: 30), lo cual pretendía entregarle dinamismo al sistema mediante la reestructuración de las funciones de las agencias ministeriales, y así, superar la parcelación burocrática e institucional del sistema educacional. Asimismo, el proyecto consultaba la creación de cinco escuelas zonales de pedagogía para la preparación del magisterio, las cuales unificarían y centralizarían "la forma y perfeccionamiento del profesorado necesario a los diversos establecimientos educacionales de la región por medio de estudios generales comunes y de las especializaciones correspondientes a cada enseñanza" (Troncoso y Navea, 1947: 31).

En el proyecto, la formación del profesorado quedaba exclusivamente en manos del Estado, por lo que las escuelas zonales fueron dependientes académica y administrativamente de la Universidad de Chile; dos de éstas, ubicadas en la primera zona norte y en la última zona del extremo sur del país, tendrían carácter internacional, con la finalidad de favorecer la integración americana, y así, "crear y mantener una verdadera solidaridad" con los países del continente.

En este contexto, las distintas resoluciones adoptadas tanto en el IV Congreso Americano de Maestros en 1943 como en el Primer Congreso Nacional de Educación de 1945 (organizado por la Federación de Educadores de Chile —FEDECH - integrada por docentes que trabajaban en liceos, escuelas primarias o en la enseñanza técnico-profesional) evidencian la profunda adhesión de los profesores al proyecto de unificación. Según lo expuesto en las actas de las sesiones de estos encuentros, durante la década de 1940, los líderes magisteriales plantearon la implementación de dos estrategias para profesionalizar el trabajo docente y la preparación pedagógica de los maestros: una medida de corto plazo, que contemplaría la unificación de criterios en la formación de profesores de las distintas instituciones educativas; en tanto que, a largo plazo, se pondría en marcha una única escuela que prepararía profesionalmente a docentes. De acuerdo con lo señalado en la Revista de Educación, una de las principales resoluciones del Congreso Nacional referido fue establecer que "mientras se va a la creación de la Escuela Única de Educación, es absolutamente necesario fijar, a los 
actuales institutos formadores del profesorado secundario, un marcado carácter profesionalpedagógico" (1945: 121).

Si bien durante la década de 1940 el proyecto presentado por el ministro, Claro Velasco, y apoyado por los pedagogos socialistas, y un posterior proyecto de transformación estructural promovido por la FEDECH ${ }^{11}$ no tuvieron éxito, debido a que en el Congreso triunfó un proyecto de reforma de carácter gradual y experimental, compuesto por el Plan de Renovación Gradual de la Enseñanza Secundaria y el Plan Experimental de Educación Rural de San Carlos, (Núñez, 1989); el líder gremial, Víctor Troncoso, continuó impulsando en los años siguientes la idea de establecer una institución que formara profesores de manera exclusiva. Es interesante notar que las distintas propuestas de unificación lideradas por este educador durante la primera mitad del siglo Xx mantienen las bases y los argumentos del primer proyecto; por ejemplo, en el contexto del Movimiento de Escuelas Consolidadas y su expansión en el sistema educativo nacional durante la década de 1950, los profesores Víctor Troncoso y Juan Sandoval (1954: 81) - a través de la publicación de La Consolidación de la Educación Pública ${ }^{12}$ - insistieron en la creación de una Escuela Única de Pedagogía que pudiera

equipar a los maestros con una base cultural y profesional uniforme [...] [la cual] termina con el anacronismo de tener diversas escuelas para formar diversos tipos de maestros que constituirán siempre el muro de contención para toda renovación de la Educación Pública; Crea en el magisterio el sentido y el espíritu de cuerpo [...]; Crea en los maestros la capacidad para expresar sus actitudes especiales [...] [y] que es más económica para el Estado y menos onerosa para sus alumnos.

Al igual que en el proyecto de Ley Orgánica de Servicios Educacionales elaborada en 1944, la propuesta replanteaba la creación de una Escuela Única de Pedagogía, la cual se presentaba como solución a la segmentación del sistema educativo, al señalar que "la posición del maestro que aprecia y siente la educación como función de Estado es el mejor instrumento para combatir el criterio de 'parceleros'” (Troncoso y Sandoval, 1954: 83). De esta manera, se sostenía que la Escuela Única de Pedagogía sería el "primer organismo del proceso de consolidación de la enseñanza, el que lo impulse y lo fortalezca por intermedio de los maestros que forme" (Troncoso y Sandoval, 1954: 81).

\footnotetext{
11 Si bien la FEDECH apoyó la idea de renovar gradualmente la enseñanza secundaria, siempre reclamó una reforma de carácter estructural, que armonizara "nuestro sistema de enseñanza con las necesidades del país" (Núñez, 2003: 77).

12 Este movimiento fue una propuesta de transformación global de la educación desarrollada en la década de 1950. Si bien se ejecutó bajo los marcos gubernamentales de las presidencias de Gabriel González Videla y Carlos Ibáñez del Campo, Iván Núñez sostiene que el movimiento de Consolidación no fue producto de la voluntad de un gobierno, sino una empresa atípica, ya que tuvo características de movimiento social con origen estatal. La Consolidación de la Educación Pública se presentó como una política educacional de carácter general, "que interpretaba la realidad del sistema educacional en su conjunto y ofrecía soluciones de alcance integral” (Núñez, 1989: 57).
} 
La unificación de la preparación profesional de los profesores se realizaría mediante la fusión en una sola institución de las 13 escuelas normales existentes en ese momento en el país, el Instituto Pedagógico y el Instituto Pedagógico Técnico. Esta nueva institución de formación docente estaría presente en distintas zonas geográficas del país, ya que la propuesta de los profesores Troncoso y Sandoval (1954: 81 y 82) dividía el territorio nacional en zonas geográfico-económicas, en las cuales funcionarían" tantas Escuelas Únicas de Pedagogía como sean necesarias".

A pesar de las dos propuestas elaboradas, principalmente, por Víctor Troncoso durante las décadas de 1940 y 1950, el proyecto de escuela única no se concretó; sin embargo, años después, los gremios magisteriales volvieron a plantear la posibilidad de implementarlo tal como se presentará en la siguiente sección.

\section{La Escuela Única de Pedagogía: demandas y debates en el contexto de reforma educacional, 1964-1970}

Un tercer intento por establecer la Escuela Única de Pedagogía se evidencia en el proceso de reforma educacional implementada durante el gobierno de Eduardo Frei Montalva entre 1964 y 1970.13 En este nuevo esfuerzo, destaca el papel desempeñado por la Escuela de Educación de la Universidad de Concepción, institución que impulsó el debate y permitió la discusión amplia respecto a la posibilidad de lograr establecer la unificación del sistema de formación de profesores.

La realización de las Jornadas de Estudio sobre la formación de profesores y la Escuela Única de Pedagogía, entre los días 10 y 11 de octubre de 1969 en Concepción, reunió a importantes actores educativos del país a discutir sobre la formación de maestros. Entre los expositores destacaban las autoridades de la Universidad de Concepción, como el rector, Edgardo Enríquez, y el decano de la Escuela de Educación, Julio Inostroza. De los organismos oficiales asistieron el ministro de educación, Máximo Pacheco; el subsecretario de educación, Ernesto Livacic; la directora general de educación primaria, Renée Viñas; el director

\footnotetext{
${ }^{13}$ La reforma educacional iniciada en noviembre de 1964 tuvo como ejes principales la universalización de la educación primaria y el mejoramiento cualitativo de la enseñanza. Para lograr estos propósitos, el gobierno demócrata cristiano implementó una serie de cambios en el sistema educativo, tales como una reforma curricular para la educación primaria y secundaria, inspirada en los principios pedagógicos de la taxonomía de objetivos de la educación de Benjamin Bloom; la ampliación de la matrícula escolar; el mejoramiento de las condiciones laborales del profesorado; la aplicación de programas de asistencia escolar; la aplicación de un nuevo modelo de formación de profesores, a través de la formación acelerada de maestros (para suplir el déficit de docentes); y el perfeccionamiento en ejercicio -respecto a este último punto, conviene destacar que el periodo que inició la reforma de la escuela normal al establecerse que la formación normalista fue el del nivel postsecundario; transformación que se interrumpió por problemas con el financiamiento del programa, como se explicará más adelante; al respecto, véase Bellei y Pérez (2016).
} 
de educación secundaria, Alfonso Bravo; y el director general de educación profesional, Luis Oyarzún. En representación de la Universidad de Chile, expusieron las profesoras Rebeca Soltanovich, Olga Poblete y Aída Parada. Por otro lado, como representantes de las organizaciones gremiales, participaron los profesores Humberto Elgueta (Federación de Educadores de Chile) y Jorge Espinoza (Sociedad Nacional de Profesores). Asimismo, comentó la propuesta, el Centro de Estudiantes de la Escuela de Educación de la Universidad de Concepción; mientras que se presentó la investigación realizada por Jiri Kotásek, director adjunto del Instituto de Formación de Profesores de la Universidad Carolina de Praga, relativa a tendencias y problemas actuales de la formación de profesores. Del mismo modo, se expuso el Informe Final sobre Formación de Personal Docente de Enseñanza Primaria y Secundaria General, elaborado por el Comité de Expertos de la UNESCO, entre los días 4 al 15 de diciembre de 1967 en París.

Durante los dos días que duraron las jornadas, se diagnosticó y discutió el diseño de un nuevo modelo de formación de profesores. A diferencia de los proyectos promovidos por Víctor Troncoso décadas antes, en las jornadas se consensuó que no se crearía un sistema completamente nuevo a partir de la disolución de las antiguas instituciones y la fundación de una nueva, sino que se unificarían los criterios comunes de acción en el corto, mediano y largo plazo "de los organismos oficiales, como los institutos formadores, [de] las organizaciones gremiales del magisterio y [de] las organizaciones estudiantiles" (Escuela de Educación, 1969: 1); de esta manera, se coordinaría la formación de todos los profesores y se proveería al sistema escolar docentes cualitativa y cuantitativamente mejor preparados para satisfacer las necesidades del país.

El análisis de las actas de las jornadas permite evidenciar un avance sustantivo en el logro de consensos de los principales actores comprometidos con el proyecto de unificación. En términos generales, y de acuerdo con las propuestas que presentaron las distintas comisiones participantes del encuentro, se consideró la Escuela Única de Pedagogía como un proceso de formación articulada y continua, y no como un establecimiento único; un criterio unívoco a todas las categorías de docentes, que suprimiría las diferencias entre maestros para la educación básica (primaria) y profesores para la enseñanza media (secundaria); una formación integrada, en donde la teoría estuviera fundamentada en la praxis docente; ${ }^{14}$ una institución que coordinaría la profesionalización del trabajo docente; un sistema que entregaría una formación común a todos los estudiantes en los primeros años de cursada, pero que después los especializaría en función de las necesidades de los niveles educativos; un espacio que configuraría y fortalecería la identidad docente, revalorizando la importante función social del profesorado.

\footnotetext{
${ }^{14}$ En este marco, las escuelas de enseñanza primaria y secundaria funcionarían como "laboratorios pedagógicos", en los cuales los aprendices practicarían la docencia.
} 
No obstante, nuevamente $-\mathrm{y}$ a pesar de los esfuerzos y por convocar a autoridades, docentes, gremios magisteriales y estudiantes - no logró concretarse completamente la idea de unificar la formación de profesores de manera sistémica. Es posible que algunas de las razones que dan cuenta del fracaso de la iniciativa se relacionen con las polémicas generadas en torno a los cambios propuestos por el gobierno demócrata cristiano en materia de formación de profesores en el periodo, los cuales se orientaban a suprimir o transformar la enseñanza normal y profesionalizar la labor docente de los maestros de primaria. La discusión en el Congreso Nacional respecto a la implementación del Programa de Desarrollo de la Enseñanza Normal, propuesto en 1967 y tramitado hasta 1970, provocó innumerables resistencias en la clase política y en los gremios docentes, puesto que el proyecto de reforma al sistema de formación de profesores normalistas fue financiado por la fundación Ford. En un contexto de guerra fría y en un clima de confrontación local de discursos antiimperialistas, el proyecto see bloqueó más por razones políticas, que educativas (Cox, 1984; Cox y Gysling, 1990).

De esta manera, el debate en torno al proyecto de unificación de la formación de profesores se desplazó, limitándose a ser discutido internamente en escuelas normales y universidades; sin embargo, la idea tomó fuerza nuevamente durante el gobierno de Salvador Allende, en el marco del proyecto de democratización de la educación pública.

\section{La Escuela Nacional Unificada y la formación de profesores, 1970-1973}

El último intento por unificar democráticamente la formación de profesores - y el más claro, por ser presentado como elemento fundamental en el proyecto— ${ }^{15}$ se encuentra en el Informe de Escuela Nacional Unificada. ${ }^{16}$ Continuando con la "tradición unificadora" de la educación pública chilena, y haciéndose cargo de las recomendaciones del Informe Faure de la UNESCO (Núñez, 2003), el gobierno de la Unidad Popular propuso la formación unificada de las nuevas generaciones de maestros como requisito para poner en práctica la Escuela Única. De acuerdo con el Informe, "si en el futuro la escuela será una sola, es lógico pensar que la formación del maestro deberá ser también una. Esta medida de unificación de la formación

${ }^{15}$ Conviene destacar que la formación del profesorado de manera unificada estuvo presente en la mayoría de las comisiones participantes en el Congreso Nacional de Educación, realizado en 1971; al respecto, se recomienda revisar los números 36, 37 y 38 de la Revista de Educación, en donde se transcriben los informes de las referidas comisiones

${ }^{16}$ Fue el proyecto de transformación integral del sistema educativo promovido por el gobierno de la Unidad Popular, coalición política liderada por el médico socialista, Salvador Allende; éste se elaboró a partir de un largo debate desarrollado en 1971, el cual involucró a actores como estudiantes, profesores, padres y apoderados, organizaciones sociales, entre otros. El principal fundamento político-ideológico del proyecto era la construcción de un sistema educacional que permitiera la transición al socialismo; en cuanto a las ideas pedagógicas, su base era el concepto de 'educación permanente'". 
del maestro tendrá también una repercusión social y profesional al desaparecer las odiosas categorías con sus consecuencias de desniveles de rentas y de status" (Ministerio de Educación Pública, 1973: 17).

En este marco, conviene señalar que durante el gobierno del presidente, Salvador Allende, el proyecto tomó un cariz diferente, porque se vinculó la formación unificada directamente con el mejoramiento de la situación económica y laboral de los educadores. La jornada de trabajo se redujo a 36 horas semanales; de las cuales, no más de 24 o 26 eran de clases y las restantes se dedicaron a labores técnicas de administración y comunidad; además, se promovieron fuertemente los "años de estudios pedagógicos y de perfeccionamiento" (Allende, 1971: 8).

El desenlace histórico de esta coyuntura educativa es conocido: el golpe de Estado de septiembre de 1973 sepultó abruptamente el último intento de unificación de la educación pública; sin embargo, y siguiendo lo planteado por Iván Núñez, "tan vivo estuvo el Estado Docente que tampoco lo mató de inmediato la dictadura militar" (2003: 65). Paradójicamente, la impronta estatista de los contraalmirantes, quienes lideraron el Ministerio de Educación entre 1973 y 1978, permitiría, por algunos años y por la fuerza, la aplicación de un sistema único de formación de docentes, tal como se evidenciará en el siguiente apartado.

\section{Crisis y unificación: la formación de profesores durante la dictadura cívico-militar, 1973-1990}

La dictadura cívico-militar liderada por el general, Augusto Pinochet, entre 1973 y 1990, transformó el sistema de formación de profesores existente en el país. La politización de las escuelas normales y el giro que se pretendió imprimir en el sistema de educación pública implicaron que, a pocos días de la irrupción política de militares en el gobierno, se iniciara un proceso de intervención de las instituciones formadoras de maestros.

Mediante la publicación del Decreto Ley Nº 179 el 10 de diciembre de 1973, la Junta Militar ordenó suspender las clases en las escuelas normales fiscales, mas no en las particulares, y las declaró en estado de reorganización, debido a la necesidad de reestructurar administrativamente el sistema de formación de profesores y la "situación de anarquía en que se desenvuelve la Enseñanza Normal, tanto en sus aspectos técnicos, administrativos y pedagógicos, y que es el propósito de la Junta de Cobierno restablecer los principios de orden, disciplina y moralidad en los establecimientos educacionales, máxime en aquellos que tienen por misión preparar el profesorado de las escuelas de enseñanza básica" (Gimeno, 2013: 120). 
Para lograr estos cometidos, se decretó la creación de Comisiones Examinadoras de alumnos, las cuales se encargarían de estudiar la idoneidad de postulantes a la docencia y controlar la infiltración marxista en las escuelas de formación del profesorado, así como la fundación de una Comisión Coordinadora Central, con la finalidad de estudiar propuestas para fundar un nuevo sistema. De acuerdo con el Decreto, las propuestas debían considerar "la denominación de la enseñanza, el número, la calidad, ubicación y estructura de los establecimientos educacionales que la imparten; los requisitos de ingreso, evaluación, promoción de alumnos, los títulos y grados que se otorguen; la reestructuración y dependencia de la mencionada enseñanza; las calidades que debe reunir el personal que en ella labore y los planes y programas a que deberá ceñirse" (Decreto N 179, 1973: 1).

Al respecto, es importante señalar que la publicación de la Directiva para la Educación en diciembre de 1973, formulada por el ministro de educación, Hugo Castro Jiménez, por orden de la Junta de Gobierno, confirmó la unificación del sistema de formación de profesores, pero no el nivel universitario de la profesión docente ${ }^{17}$. De acuerdo con el documento, se configuraría un nuevo sistema de educación superior, en el cual los institutos pedagógicos se encargarían de "preparar a todos los profesores de párvulos, básica y media [...] Naturalmente, el quehacer académico y el contenido de los Programas de los Institutos Pedagógicos deben estar estrechamente vinculados con las necesidades y la Orientación de la Enseñanza que imparta el Ministerio de Educación, de manera que sus profesionales graduados sirvan a la actividad que los espera" (Ministerio de Educación Pública, Directiva, 1973: 1).

A pesar de las incongruencias planteadas por la Directiva en diciembre de 1973, en marzo de 1974, la formación de profesores pasó a tener nivel universitario. La publicación del Decreto Ley $N^{\circ} 353$, que recogía las conclusiones de un informe elaborado por la Comisión Coordinadora Central -el cual planteaba la urgencia de consolidar un sistema unificado de formación de docentes - traspasó de forma exclusiva a las universidades la responsabilidad de preparar profesionalmente a profesores y otorgar títulos "en un Sistema Nacional de Formación de Docentes" (Decreto N³53, 1974: 1), y estableció la organización de un Consejo de Coordinación Nacional de Formación de Docentes, bajo dependencia del Consejo de Rectores. Paradójicamente, la coordinación de un sistema de formación unificada era impuesta de manera forzada, en el contexto de un proceso de racionalización de la estructura estatal; sin embargo, este sistema se modificó en 1981.

La publicación de la Ley General de Universidades reestructuró nuevamente el sistema de formación de profesores y revirtió lo dispuesto en 1974: las pedagogías no eran parte de

\footnotetext{
${ }^{17}$ Este planteamiento fue duramente criticado por Santiago Quer Antich (1974: 10), profesor de la Escuela de Educación de la Universidad Católica de Chile, "Entre los educadores no habría necesidad siquiera de señalar los inconvenientes de todo tipo para la formación de educadores que tiene la resurrección de institutos pedagógicos, que significaron la formación de especialistas pero no de educadores y que siempre supeditaron la educación a la especialización".
} 
las 12 profesiones exclusivamente universitarias, ${ }^{18}$ a pesar de que se reconocía la "trascendencia social y moral" (Consejo de Rectores de Universidades Chilenas, 1981: 41) del educador; de esta manera, los institutos profesionales podrían impartir carreras de educación. Incluso, mediante la promulgación de un Decreto con Fuerza de Ley, las sedes Valparaíso y Santiago de la Facultad de Educación de la Universidad de Chile se convirtieron en institutos profesionales, denominándose Academia Superior de Ciencias Pedagógicas de Santiago y Valparaíso. ${ }^{19}$

No obstante, con la publicación de la Ley Orgánica Constitucional de Enseñanza, la pedagogía volvió a ser reconocida como carrera exclusivamente universitaria. De acuerdo con el artículo $52^{\circ}$, los títulos de profesor de educación básica, media y diferencial requerirían el grado de licenciado (Ley $N^{\circ}$ 18.962, 1990); pero, la ley mantenía la facultad de aquellos institutos profesionales creados con base en los artículos transitorios de los Decretos con Fuerza de Ley $N^{\circ} 1$ y N 5 de 1981 de continuar impartiendo carreras de pedagogía. Esta facultad sólo se limitó en junio de 2014, cuando la Contraloría General de la República resolvió que, con la entrada en vigencia de la Ley General de Educación (Ley N²0.370, 2009), los institutos profesionales no podrían dictar carreras que necesitaran el grado de licenciatura previa, como es el caso de las pedagogías.

A pesar de los intentos señalados, no se logró concretar el proyecto de creación de un sistema unificado de formación de profesores en Chile. Si bien el cierre de las escuelas normales durante la dictadura cívico-militar significó concentrar la formación docente en el nivel universitario, las fuentes muestran que la unificación no se consiguió como tal, puesto que las instituciones no acordaron unificar currículos ni establecer criterios comunes de formación, tampoco establecieron los requisitos de ingreso o estándares de calidad. Sumado este punto a la posibilidad de que institutos profesionales impartieran pedagogías, la formación de profesores durante las décadas siguientes se vio fuertemente precarizada y segmentada institucional y socialmente.

\footnotetext{
${ }^{18}$ Las autoridades argumentaron que debían estudiar con mayor detalle el nombramiento de la pedagogía como carrera exclusivamente universitaria (debido a su importancia social, debía tener otro estatus), razón por la cual no sería parte del primer listado publicado en la Ley General de Universidades.

${ }^{19}$ Decreto con Fuerza de Ley Nº 7, 17 de febrero de 1981. Posteriormente, en 1985, se les devolvió el estatus universitario a las academias superiores de ciencias pedagógicas, creándose la Universidad Metropolitana de Ciencias de la Educación y la Universidad de Playa Ancha de Ciencias de la Educación mediante las leyes N 18.434 y 18.433, respectivamente.
} 


\section{Conclusión}

Con base en lo expuesto a lo largo de este trabajo, es posible sostener que los intentos de profesionalización y formación unificada del profesorado estuvieron presentes en todos los proyectos de reforma educativa propuestos durante el siglo xx, presentándose como factores fundamentales en todos los planes de cambio y mejoramiento de la educación.

Inicialmente, la idea de unificar la formación de docentes se originó en el contexto de elaboración del proyecto de reforma educacional por parte de la Asociación General de Profesores, que fue implementado entre diciembre de 1927 y septiembre de 1928. Tal como se explicó en la primera parte de este artículo, la aplicación de la propuesta de reestructuración institucional de la formación de profesores sólo duró nueve meses, y tuvo como desenlace la suspensión de la ejecución del proyecto y el inicio de una contrarreforma educativa. Posteriormente, la idea de unificación de la formación de profesores en Chile permaneció latente en el ideario pedagógico de los líderes magisteriales de la reforma educativa de 1928 hasta la década de 1940, año en el cual se consolidó la aspiración de los profesores Víctor Troncoso, Daniel Navea y Juan Sandoval en la propuesta de Ley Orgánica de Servicios Educacionales. Un tercer intento fue defendido transversalmente por maestros, profesores secundarios, docentes universitarios y autoridades gubernamentales en el marco de la reforma educativa iniciada en 1964. Al igual que el proyecto de 1928, la propuesta elaborada en el quinquenio 1965-1970 planteaba la transformación radical de las escuelas normales, lo cual despertó gran resistencia por parte de diversos grupos. Finalmente, y de manera paradójica, el proyecto se instrumentó por la fuerza en 1973 (sólo por algunos años, y de forma limitada, únicamente en cuanto a la concentración de la formación docente en el nivel universitario), en el contexto de la dictadura de Augusto Pinochet.

Todas estas propuestas coincidieron en la necesidad de reestructurar institucionalmente el sistema de formación de docentes, solucionar la segmentación y la parcelación de la educación pública y de los profesores primarios y secundarios, unificar los criterios curriculares de las carreras de pedagogía, fortalecer la identidad y mejorar la labor docente.

Esta mirada histórica a la formación de profesores plantea más preguntas que respuestas. A casi 90 años del primer intento de unificación de formación profesional de maestros en Chile, se hace necesario profundizar en algunos elementos de los proyectos analizados en este artículo, tales como las influencias trasnacionales recibidas por los protagonistas de los procesos de reforma, las razones que están detrás del abandono o rescate de algunas propuestas en las distintas etapas estudiadas (por ejemplo, el énfasis en las mejoras salariales en determinados períodos o la demanda de elaborar un currículum con criterios comunes), o los cambios implementados en materia de formación docente con posterioridad a la apli- 
cación de la Ley Orgánica Constitucional de Enseñanza en 1990. Se espera profundizar estas problemáticas en próximos artículos de investigación.

\section{Fuentes}

\section{Documentos}

Ministerio de Educación Pública (1927), "Decreto N 7.500, 'Reforma Educacional'”, 10 de diciembre de 1927, Santiago, Chile.

Ministerio de Educación Pública (1928a), "Decreto № 332, 'Reglamento de las Escuelas Normales', 14 de febrero de 1928, Santiago, Chile.

Ministerio de Educación Pública (1928b), "Decreto №5.280, 'Reorganización de las Escuelas Normales"”, 29 de octubre de 1928, Santiago, Chile.

Ministerio de Educación Pública (1928c), "Decreto N 5.857, 'Organización de la Enseñanza Normal'”, 17 de diciembre de 1928, Santiago, Chile.

Ministerio de Educación Pública (1932), "Decreto N²20, 'Reorganiza los servicios de educación"', 22 de julio de 1932, Santiago, Chile.

Ministerio de Educación Pública (1973a), Decreto con Fuerza de Ley Nº 179, "Declara en reorganización la Enseñanza Normal chilena", 13 de diciembre de 1973, Santiago, Chile, documento disponible en: $<$ https://www.leychile.cl/N?i=215136\&f=1973-12-13\&p=>

Ministerio de Educación Pública (1973b), "Directiva Nº 87 para la educación", 19 de diciembre de 1973, Santiago, Chile.

Ministerio de Educación Pública (1974), "Decreto N 353, 'Fija normas sobre sistema de formación docente y dispone medidas relativas a las escuelas normales del país"', 11 de marzo de 1974, Santiago, Chile, documento disponible en: <https://www.leychile.cl/N?i=6010\&f=1974-03-15\&p=>.

Ministerio de Educación Pública (1981), "Decreto con Fuerza de Ley Nº 7, 17 de febrero de 1981", Santiago, Chile, documento disponible en: <https://www.leychile.cl/Navegar?idNorma=3750>

Ministerio de Educación (1990), Ley N 18.962, "Ley Orgánica Constitucional de Enseñanza", Diario Oficial de Chile, 10 de marzo de 1990, Santiago, Chile, documento disponible en: <https://www. leychile.cl/Navegar?idNorma=30330

Ministerio de Educación (2009), Ley N²0.370, "Ley General de Educación”, 12 de septiembre de 2009, Santiago, Chile, documento disponible en: <http://bcn.cl/lzndc>.

\section{Bibliografía}

Ahumada, Jorge (1966), La crisis integral de Chile, Editorial Universitaria, Santiago. (1958), En vez de la miseria, Editorial del Pacífico, Santiago.

Allende, Salvador (1971), "Del Presidente Allende a los profesores y alumnos", Revista de Educación, núm. 34, marzo, pp. 2-10.

Bellei, Cristián y Pérez, Camila (2016), "Democratizar y tecnificar la educación. La reforma educacional de Eduardo Frei Montalva", en C. Huneeus y J. Couso (eds.), Eduardo Frei Montalva: un gobierno reformista. A 50 años de la 'Revolución en Libertad', Santiago, Editorial Universitaria.

Consejo de Rectores de Universidades Chilenas (1981), Nueva legislación universitaria chilena, Secretaría General del Consejo de Rectores, Santiago.

Cox, Cristián (1986), Continuity, conflict and change in state education in Chile: a study of the pedagogic projects of the Christian Democrat and the Popular Unity Governments, University of London.

Cristián y Jacqueline Gysling (1990), La formación del profesorado en Chile, 1842-1990, CIDE, Santiago. 
Escuela de Educación, Universidad de Concepción (1969), Jornadas de Estudio sobre la formación de profesores y la Escuela Única de Pedagogía, Ciudad Universitaria, Concepción.

Galdames, Luis (1913), La gratuidad de la educación, s.n., Imprenta, Litografía i Encuadernación Barcelona, Santiago [N. del E.: documento pdf disponible en: <http://www.memoriachilena.cl/archivos2/pdfs/ MC0057570.pdf> (fecha de consulta: 1/6/17)].

(1912), Educación económica e intelectual, Imprenta Universitaria, Santiago [N. Del E.: documento pdf disponible en: <http://www.memoriachilena.cl/archivos2/pdfs/mc0018478.pdf> (fecha de consulta: 1/6/17)].

Gimeno, Miguel (2013), "Cierre de las Escuelas Normales en Chile (1973-1974). Decretos N 179 y 353", Cuadernos Chilenos de Historia de la Educación, núm. 2, pp. 11-121.

Hamuy, Eduardo (1961), El problema educacional del pueblo de Chile, Editorial del Pacífico, Santiago. (1960), Educación elemental, analfabetismo y desarrollo económico, Editorial Universitaria, Santiago. Núñez, Iván (2003), La ENU entre dos siglos: ensayo histórico sobre la Escuela Nacional Unificada, LOM, Santiago

(2002), "La formación de docentes. Notas históricas", en Beatrice Ávalos, Profesores para Chile, historia de un proyecto, Ministerio de Educación, Santiago.

(1989), La descentralización y las reformas educacionales en Chile. 1940-1973, PIIE, Santiago.

(1987), El trabajo docente. Dos propuestas históricas, PIIE, Santiago.

(1986), Gremios del Magisterio. Setenta años de Historia, PIIE, Santiago.

Reyes Jedilicki, Leonora (2010), "Movimiento educacional, crisis educativa y reforma de 1928", Docencia, núm. 40, mayo, pp. 40-49.

Salas, Darío (1917), El problema nacional, Bases para la reconstrucción de nuestro sistema escolar primario, Sociedad Imprenta y Litográfica "Universo", Santiago.

(1913), Sobre educación popular, Imprenta Universitaria, Santiago.

Salgado, Ramón (2006), La formación docente en la región: de las normales a las universidades, UNESCO, Caracas.

Quer Antich, Santiago (1974), Análisis crítico de la Directiva para la Educación, Escuela de Educación, Universidad Católica de Chile, Santiago.

Serrano, Sol, Francisca Rengifo y Macarena Ponce de León (2013), Historia de la educación en Chile (18102010). Tomo Il: La educación nacional (1880-1930), Taurus, Santiago.

Troncoso, Víctor (1927), Igualdad social y económica del magisterio, La Nación, Santiago.

y Daniel Navea (1947), Chile reconstruye su educación: anteproyecto de la ley orgánica, Imprenta Cultura, Santiago.

Troncoso, Víctor; y Juan Sandoval (1954), Consolidación de la educación pública, Imprenta Germinal, Santiago

Zemelman, Myriam (2010), "La experimentación y sus obstáculos: Plan de Renovación Gradual de la Enseñanza Secundaria y Plan San Carlos", Revista Docencia, núm. 40, pp. 50-58.

Camila Pérez-Navarro. Docente en la Facultad de Educación, Pontificia Universidad Católica de Chile. Candidata a doctora en educación. Líneas de investigación: historia de la educación durante la segunda mitad del siglo xx chileno, específicamente, durante el gobierno de Eduardo Frei Montalva (1964-1970) y la dictadura cívico-militar chilena (1973-1990). Publicaciones recientes: "Democratizar y tecnificar la educación. La reforma educacional de Eduardo Frei Montalva”, en Carlos Huneeus y Javier Couso (eds.), (2016), Eduardo Frei 
Montalva: un gobierno reformista. A 50 años de la "Revolución en Libertad", Editorial Universitaria, Santiago, pp. 207-244 (en coautoría con Cristián Bellei); y "Es nuestra escuela de brazos abiertos". Historia de la Escuela Experimental de Niñas República del Ecuador, Santiago (en prensa; en coautoría con Claudia Drago).

Recibido: 18 de diciembre de 2016.

Aceptado:31 de marzo de 2017 